\title{
Prevalence of Blomia tropicalis allergy in two regions of South Africa
}

\author{
A C Jeevarathnum, ${ }^{1}$ MB BCh, FCPaed (SA), Dip Allerg (SA), MMed (Paed); A van Niekerk, ${ }^{1}$ MB ChB, MMed (Paed); \\ R J Green, ${ }^{1}$ MB BCh, DCH (SA), FCPaed (SA), MMed (Paed), FCCP, PhD, Dip Allergy (SA), FAAAAI, FRCP, DSc; \\ P Becker, ${ }^{2}$ BSc Hons, MSc, PhD; R Masekela, ${ }^{3}$ MB BCh, MMed (Paed), Dip Allerg (SA), Cert Pulmonol (SA) Paed, FCCP, PhD

\begin{abstract}
${ }^{1}$ Department of Paediatrics and Child Health, Division of Pulmonology, Faculty of Health Sciences, University of Pretoria, South Africa
${ }^{2}$ Faculty of Health Sciences, University of Pretoria, South Africa

${ }^{3}$ Department of Maternal and Child Health, School of Clinical Medicine, College of Health Sciences, Nelson R Mandela School of Medicine, University of KwaZulu-Natal, Durban, South Africa
\end{abstract}

Corresponding author: R Masekela (masekelar@ukzn.ac.za)

\begin{abstract}
Background. Asthma and allergic rhinitis affect $15 \%$ and $38 \%$ of South African (SA) children, respectively. The housedust mite (HDM) is the most significant indoor aeroallergen. Typical HDM species include Dermatophagoides pteronyssinus, D. farinae and Blomia tropicalis. Conventional skin-prick testing (SPT) panels only test for Dermatophagoides. B. tropicalis has been described in the tropical and subtropical regions, but is not routinely tested for in SA.

Objective. To ascertain the significance of B. tropicalis as an aeroallergen in northern coastal KwaZulu-Natal Province (KZN), a tropical environment, and in Johannesburg in the highveld, where the climate is milder and less humid.

Methods. Children aged $1-18$ years with features of allergic rhinitis and/or asthma were recruited over a 6-month period from Alberlito Hospital in northern KZN and the Clinton Clinic in Johannesburg. SPTs included Dermatophagoides and B. tropicalis. Sensitisation was defined as a wheal $3 \mathrm{~mm}$ greater than the negative control.

Results. Eighty-five subjects were included, 50 in northern KZN and 35 in the Johannesburg arm; $52 \%$ of subjects in northern KZN and $3 \%$ in Johannesburg were sensitised to B. tropicalis, with a significant difference between these centres $(p<0.05)$. Of the $52 \%$ sensitised to $B$. tropicalis in northern KZN, half were sensitised only to $B$. tropicalis.

Conclusion. There is a high prevalence of B. tropicalis allergy in the tropical northern KZN region and a much lower prevalence in the Johannesburg region. Routine testing for B. tropicalis allergy should be employed in northern KZN.
\end{abstract}

S Afr Med J 2015;105(7):567-569. DOI:10.7196/SAMJnew.7786

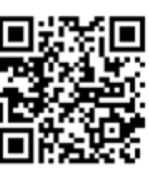

Atopy and allergic disease form part of an expanding field of medicine and include conditions such as asthma, allergic rhinitis, allergic conjunctivitis, atopic eczema and food and drug allergies, to name just a few.

The prevalences of allergic rhinitis and asthma among South African (SA) children are as high as $38.5 \%$ and $15 \%$, respectively. ${ }^{[1,2]}$ Asthma and allergic rhinitis are closely linked, and physicians are well aware of this link in the form of the 'united airway' theory. ${ }^{[3]}$ It has been well described that co-management of these two conditions will reduce disease severity and improve quality of life. ${ }^{[3]}$ Aeroallergens have been implicated in the pathophysiology of both these disease processes. ${ }^{[4]}$ Those implicated include housedust mite (HDM), grass, mould and cockroach, among others. However, HDM allergy is the most important indoor aeroallergen in SA, affecting allergic patients with asthma and allergic rhinitis. ${ }^{[4-6]}$ One study in the Durban region found the incidence of HDM allergy among asthmatics to be as high as $95 \% .{ }^{[7]}$

Worldwide, there are a number of HDM species involved in these atopic disease processes. These include Dermatophagoides pteronyssinus, D. farinae, Euroglyphus maynei and Blomia tropicalis. The prevalence of these mite species differs geographically. ${ }^{[8]}$ Dermatophagoides spp. occur worldwide, and prefer a warm and temperate climate with humidity of $>55 \%{ }^{[8]}$ Generally the prevalence of $D$. pteronyssinus is higher in areas with higher humidity than that required by $D$. farinae. Australia, New Zealand, the UK and Mauritius have a predominance of $D$. pteronyssinus, ${ }^{[9]}$ while $D$. farinae predominates in South Korea, Jakarta, Indonesia and Italy. ${ }^{[9]}$ E. maynei is thought to occur worldwide, but it has been poorly studied and the exact incidence and predominance are unknown ${ }^{[8]} B$. tropicalis is thought to occur most commonly in the tropical and subtropical regions of the world, such as Singapore, Malaysia, the Philippines and Hong Kong. ${ }^{[9]}$

B. tropicalis allergy can be proven via in vivo or in vitro methods. In vivo methods involve use of skinprick testing (SPT) and in vitro methods use of ImmunoCAP (ThermoFisher) assays. The SPT and ImmunoCAP test panels currently in use for the identification of HDM allergy in SA test only for Dermatophagoides spp. unless specifically requested. Dermatophagoides is therefore the most studied HDM species in SA, and data relating to the other species are poorly defined. There is limited cross-reactivity between Dermatophagoides spp. and B. tropicalis, both on SPTs in vivo and $\operatorname{IgE}$ binding on ImmunoCAP studies in vitro. ${ }^{[10-12]}$ However, there is marked crossreactivity between $D$. pteronyssinus and $D$. farinae on both tests. ${ }^{[11,12]}$ It therefore stands to reason that a positive SPT for $D$. pteronyssinus or $D$. farinae does not indicate a positive result for $B$. tropicalis, and individual testing for $B$. tropicalis is necessary to prove $B$. tropicalis allergy. Current testing practices in SA do not include B. tropicalis testing other than in the Western Cape region. ${ }^{[2]}$

Specific immunotherapy offers the only potential cure for HDM allergy. Immunotherapy can comprise either sublingual or subcutaneous therapy, both of which have been found to be effective 
in the treatment of HDM allergy. ${ }^{[2]}$ However, separate reagents for Dermatophagoides and Blomia are required. Current routine testing will not identify patients who suffer from B. tropicalis allergy, and the correct immunotherapy will therefore not be ordered for patients who need it. However, these patients can potentially be cured if a diagnosis of $B$. tropicalis allergy is made.

B. tropicalis should be individually tested for in areas of the world in which it is highly prevalent. ${ }^{[10]}$ If the parts of SA in which $B$. tropicalis is of significance can be identified, testing in these regions can be advocated.

\section{Objective}

To determine the significance of $B$. tropicalis allergy among patients with allergic rhinitis and/or asthma in two different regions of SA, namely the northern coastal region of KwaZulu-Natal province $(\mathrm{KZN})$ and the Johannesburg area. The northern KZN region is located at sea level and has a warm subtropical climate all year round. Johannesburg is situated in the highveld at $1753 \mathrm{~m}$ above sea level, and the climate is milder and dry during most of the year.

\section{Methods}

A retrospective cross-sectional study design was employed. The study was undertaken at two private paediatric practices run by AVN, one situated at the Clinton Clinic in Johannesburg and the other at Alberlito Hospital in northern KZN. All the patients who presented with features of allergic rhinitis and/or asthma and had SPTs for aeroallergens between September 2013 and February 2014 were included in the study. Only SPTs were utilised in this study to confirm sensitisation.

Two reagents were utilised for the SPTs at both centres to test specifically for HDM allergy. Both are manufactured by Stallergenes, France. The first was the conventional reagent used by most centres in SA that test for D. pteronyssinus and D. farinae allergy (D. pteronyssinus and D. farinae mix). The second was a specific reagent that tested for $B$. tropicalis allergy specifically. A positive SPT result was defined as a wheal $3 \mathrm{~mm}$ greater than the negative control.

\section{Statistical analysis}

For both B. tropicalis and D. pteronyssinus/D. farinae, data were reported by province and the proportions of positive tests along with $95 \%$ confidence intervals (CIs). Comparisons between the two settings with respect to the proportions of positive B. tropicalis and $D$. pteronyssinus/D. farinae tests, respectively, were done using Fisher's exact test. The association between allergens and site was expressed in terms of odds ratios with $95 \%$ CIs. Testing was done at the 0.05 level of significance.

\section{Ethical considerations}

Full ethical approval was obtained from the Faculty of Health Sciences Research Ethics Committee of the University of Pretoria. Consent for use of clinical data was obtained from AVN, who was the primary physician at both study sites.

\section{Results}

A total of 87 charts from the two centres were screened; two were excluded because only ImmunoCAP testing for inhaled allergens was performed and not SPT analysis. All the patients were between 1 and 18 years of age, with a male-to-female ratio of 2:1 (Table 1). The mean age of the study population was 7.4 years.

There was sensitisation to Dermatophagoides in $40.0 \%$ (95\% CI 26.4 - 54.8) of children in the Alberlito arm as opposed to 22.9\% (95\% CI 10.4 - 40.1) in the Clinton Clinic arm (Fig. 1). The

$\begin{aligned} & \text { Table 1. Characteristics of all participants with asthma and } \\
& \text { allergic rhinitis included in the study }\end{aligned}$
\begin{tabular}{lll}
\hline Variable & Johannesburg & KZN \\
\hline Patients, $n$ & 35 & 50 \\
Age (years), mean (range) & $7.4(1-18)$ & $7.4(1-15)$ \\
Gender (M/F), $n$ & $27 / 8$ & $30 / 20$ \\
Dermatophagoides $(\%)^{*}$ & 22.9 & 40.0 \\
B. tropicalis $(\%)^{*}$ & 2.9 & 52.0 \\
$* \%$ of patients with sensitisation to the specific HDM.
\end{tabular}

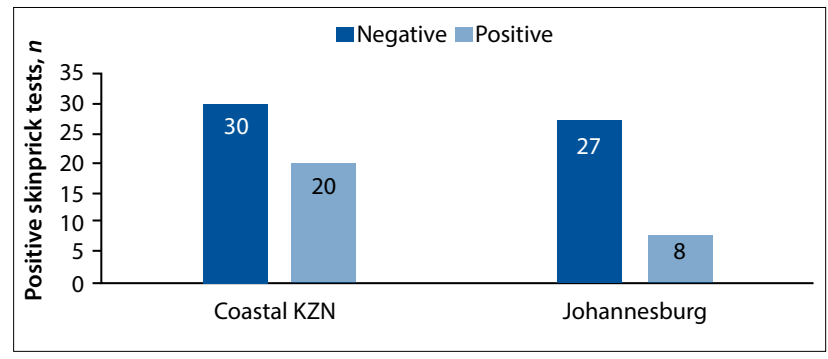

Fig. 1. A comparison between positive SPTs for Dermatophagoides spp. at Alberlito Hospital (KZN) and Clinton Clinic (Johannesburg).

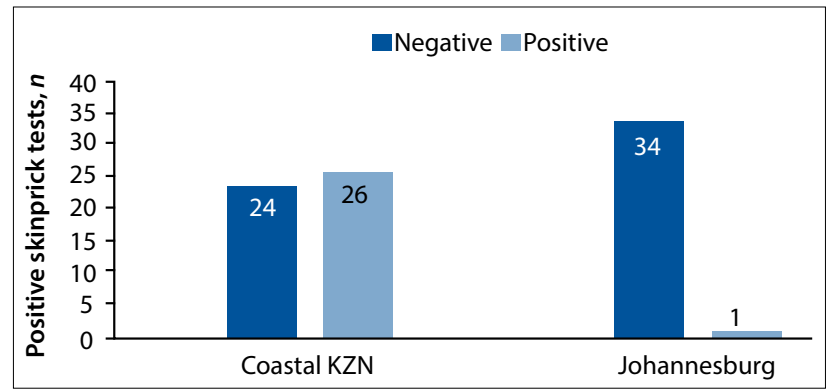

Fig. 2. A comparison between positive SPTs for $\mathrm{B}$. tropicalis at Alberlito Hospital (KZN) and Clinton Clinic (Johannesburg).

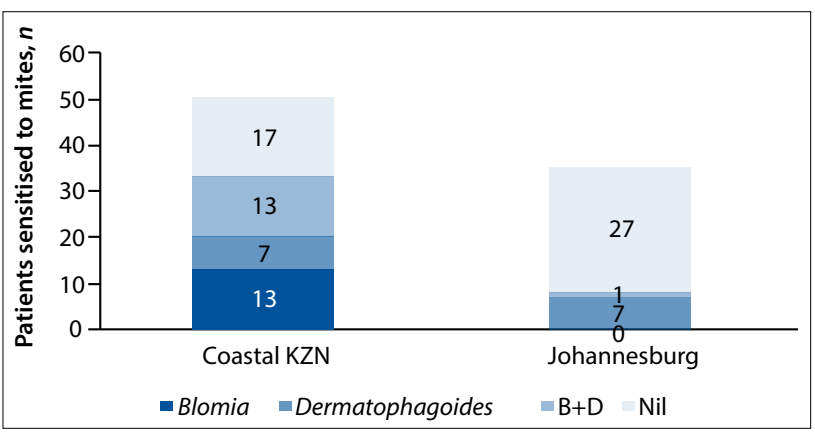

Fig. 3. A summary of sensitisation to B. tropicalis and Dermatophagoides mites according to site.

difference between the two groups was not statistically significant $(p=0.077)$. For B. tropicalis there was sensitisation in $52.0 \%(95 \%$ CI $37.4-66.3)$ of children in the Alberlito arm as opposed to 2.9\% (95\% CI 0.1 - 14.9) in the Clinton Clinic arm (Fig. 2). The difference between the two groups was statistically significant $(p<0.001)$.

On further analysis of the 33 patients in the Alberlito arm who tested positive for any HDM, 13/50 (26.0\%) tested positive for only B. tropicalis, $7 / 50$ (14.0\%) positive for only Dermatophagoides spp., and 13/50 (26.0\%) positive for both 
Blomia and Dermatophagoides (Fig. 3). In contrast, on analysis of the 8 patients in the Clinton Clinic group sensitised to any HDM, 7/35 (20.0\%) tested positive only for Dermatophagoides and $1 / 35$ (2.9\%) tested positive for B. tropicalis. The only patient who tested positive for $B$. tropicalis was also positive for Dermatophagoides.

\section{Discussion}

This study demonstrates that allergy to Dermatophagoides spp. occurs both on the subtropical northern KZN coast of SA and in the temperate highveld region, with no statistical difference between the two regions. However, the proportion of B. tropicalis sensitisation was significantly higher in northern KZN, with $52 \%$ of individuals as opposed to only $2.9 \%$ for the highveld.

Previous studies have shown a higher prevalence of HDM allergy (Dermatophagoides spp.) along the coastline of SA compared with the highveld region. ${ }^{[5,6]}$ However, the current study showed no statistical difference in the prevalence of Dermatophagoides between the KZN coast and the highveld. Reasons for the high prevalence of Dermatophagoides spp. in all regions include indoor heating in highveld houses, favouring proliferation of the mites. From this study it is evident that Dermatophagoides plays a significant role in allergic rhinitis and asthma among children both in the highveld and on the KZN coast. Guidelines should therefore require that routine testing for Dermatophagoides spp. is employed all over SA.

Around the world, B. tropicalis is abundant in the tropical and subtropical regions where there is high humidity and warm temperatures. One of the reasons Blomia is so prevalent along the KZN coastline may be the weather patterns in this region. The northern KZN area is located at sea level and has a humid climate much like that experienced in the tropical and subtropical belt. The warm Mozambique current that flows along the coast is responsible for warmer seas, warmer temperatures and higher humidity. ${ }^{[7]}$ This warm and humid climate is much like that in the subtropical belt and could explain the high prevalence of $B$. tropicalis along this coastline. A 2010 study in Mexico City, which does not have a temperate climate, showed B. tropicalis allergy to be highly prevalent in patients with allergic rhinitis and asthma. ${ }^{[13]}$ It therefore seems possible that there may be reasons in addition to humidity and temperature why Blomia may become significant in a particular area. Further studies are required to determine these reasons.

Routine testing for B. tropicalis is suggested in warm, tropical environments. ${ }^{[10]}$ There are no formal studies from SA that describe the exact incidence of this mite in different regions of the country. According to expert opinion, $40 \%$ of allergic patients in the Western Cape are estimated to be allergic to B. tropicalis. Further studies to delineate the exact importance of $B$. tropicalis in this region are under way. ${ }^{[5,6]}$ We have demonstrated that another region in SA, namely northern KZN, also has a high prevalence of HDM sensitisation (66.0\% overall). B. tropicalis prevalence is high, with $26.0 \%$ of new subjects presenting with features of allergic rhinitis or asthma being sensitised to both Blomia and Dermatophagoides and $26.0 \%$ to Blomia alone. The routine testing currently employed in SA, either by in vivo or in vitro methods, would miss these cases.

B. tropicalis requires a specific reagent during SPT analysis. This is not usually included in most SPT panels for aeroallergens done in SA. The ImmunoCAP screening assay most commonly done for inhaled allergens in SA is the Phadiatop assay. While this is an excellent screening tool for the diagnosis of aeroallergen sensitisation in SA, and includes testing for grass, trees, cockroach, cat, dog and HDM, it tests only for allergy to Dermatophagoides spp. and not to Blomia. Since neither routine SPTs nor the Phadiatop assay screen for $B$. tropicalis allergy, current testing strategies are deficient in view of our finding of a high prevalence of Blomia sensitisation in northern KZN.

One of the strategies for treatment of allergic disease is avoidance of the known allergen. However, HDM allergen avoidance is not an option because HDMs are so abundant in the indoor environment. ${ }^{[2]}$ As avoidance strategies are not possible, definitive treatment for HDM allergy, with the possibility of a cure, can be undertaken in the form of immunotherapy. Just as the diagnosis of HDM allergy differs between Dermatophagoides and Blomia, with specific regents required on SPT analysis, so too does immunotherapy for the two species differ, particular agents being required for each species. If routine testing is employed for Blomia in the northern KZN region, patients who are allergic to it can be offered the appropriate immunotherapy.

\section{Study strengths and limitations}

One of the strengths of this study is that it utilised SPT and not ImmunoCAP testing. SPT is a much more affordable option and can therefore be done on a wider scale. However, the specific reagent for Blomia should be included in highly prevalent areas.

The study is limited by the relatively small sample size and the relatively short period of time during which it was conducted. It also has geographical limitations in that it only involved two private paediatric practices in the each of the regions investigated.

\section{Recommendations}

It is recommended that more studies be undertaken to ascertain the significance of $B$. tropicalis as an aeroallergen in coastal and tropical parts of SA. This would offer a countrywide assessment of the prevalence of $B$. tropicalis and indicate those areas in which it should be routinely tested for.

\section{Conclusion}

There is a high prevalence of $B$. tropicalis allergy in tropical northern KZN and a much lower prevalence in the non-tropical Johannesburg region. Routine testing for $B$. tropicalis allergy should be employed in northern KZN.

\section{References}

1. Motala C, Green RJ, Manjra AI, Potter PC, Zar HJ. Guidelines for the management of chronic asthma in children: 2009 update. S Afr Med J 2009;99(12):898-912.

in children: 2009 update. S Afr Med J 2009;99(12):898-912.
2. Manira AI. Allergic rhinitis in children. CME 2011:29(1):25-29.

2. Manjra AI. Allergic rhinitis in children. CME 2011;29(1):25-29.
3. Green RJ, Hockman M, Friedman R, et al. Allergic rhinitis in South Africa: 2012 Guidelines. S Afr Med Green RJ, Hockman M, Friedman R, et al. Allergic rhinitis in South

4. Potter PC. Allergic evaluation and management of the atopic patient. S Afr Fam Pract 2008;50(5):1826. [http://dx.doi.org/10.1080/20786204.2008.10873755]

Potter PC. Overview of the indigenous allergens of South Africa. Curr Allergy Clin Immunol 2007;20(4):174-176

6. Potter PC, Davis G, Manjra A, Luyt D. House dust mite allergy in South Africa - historical perspective and current status. Clin Exp Allergy 1996;26(2):132-137. [http://dx.doi.org/10.1111/j.1365-2222.1996. tb00071.x

7. Potter PC. Common indoor and outdoor aero-allergens in South Africa. CME 2010;28(9):426-430.

8. Arlian LG, Morgan MS, Neal JS. Dust mite allergens: Ecology and distribution. Curr Allergy Asthma Rep 2002;2(5):401-411. [http://dx.doi.org/10.1007/s11882-002-0074-2]

Rep 2002;2(5):401-411. [http://dx.doi.org/10.1007/s11882-002 OC7-2] 224 .

10. Caraballo L, Puerta L, Matrinez B, Moreno L. Identification of allergens from the mite Blomia tropicalis. Caraballo L, Puerta L, Matrinez B, Moreno L. Identification of allergens from the mite Blomia tropicalis.
Cxp Allergy 1993;24(11):1056-1060. [http://dx.doi.org/10.1111/j.1365-2222.1994.tb02743.x]

Clin Exp Allergy 1993;24(11):1056-1060. [http://dx.doi.org/10.1111/j.1365-2222.1994.tb02743.x]
11. Chew FT, Yi FC, Chua KY, Fernandez-Caldas E, et al. Allergenic differences between the domestic 1. Chew FT, Yi FC, Chua KY, Fernandez-Caldas E, et al. Allergenic differences between the domestic
mites Blomia tropicalis and Dermatophagoides pteronyssinus. Clin Exp Allergy 1999:29(7):982-988. [http://dx.doi.org/10.1046/j.1365-2222.1999.00543.x]

12. Farnandez-Caldas, Lockey RF. Blomia tropicalis, a mite whose time has come. Allergy 2004;59(11):11611164. [http://dx.doi.org/10.1111/j.1398-9995.2004.00727.x]

13. Martinez JNE, Aquilar AD, Rojas RE. Sensitization to Blomia tropicalis and Dermatophagoides pteronyssinus, farinae and siboney prevalence in patients with rhinitis, allergic asthma, or both, in a population of a metropolitan area of Mexico City. Rev Alerg Mex 2010;57(1):3-10 